\title{
NEW THEORY OF SINGLE BUNCH STABILITY IN A LINAC WITH QUADRUPOLE DISPLACEMENTS.
}

\author{
G. Guignard, J. Hagel, CERN, Geneva, Switzerland
}

\begin{abstract}
The analytical treatment previously described [1] has been extended to include the important effect of magnetic quadrupole transverse displacements, the chromatic variation of the magnetic focusing, the energy spread along the bunch and possible microwave quadrupoles, the last two in relation to BNS damping. Both, the longitudinal and transverse equations of motion are solved, the second by using the perturbation method with partial expansions developed for this theory. The localized nature of the quadrupole displacements is preserved by using thin lenses and the superposition principle for the kick effects. The causality principle applied to the downstream beam oscillations due to the kicks is introduced via Heaviside functions. The treatment presented [2] provides formulae for the tuneshift in the bunch and first-order solutions for the transverse beam off-sets within the bunch. It presents a break-through in the recent efforts [3] to solve the problem of the bunch stability theoretically, with realistic beam and linac models.
\end{abstract}

\section{EQUATIONS OF MOTION}

The equations of motion for the longitudinal and transverse (vertical) plane in a linac with longitudinal and transverse wakefields is given in the form of two semicoupled partial and linear integro-differential equations [4] as:

$$
\begin{aligned}
& \frac{\partial \gamma(s, z)}{\partial s}=\frac{e U}{m_{0} c^{2}} \cos \left(k_{R F} z-\bar{\Phi}_{R F}\right)- \\
& -C \int_{0}^{z} \rho\left(z^{*}\right)\left[W_{L 0}+\frac{W_{L 1}-W_{L 0}}{l_{B}}\left(z-z^{*}\right)\right] d z^{*} \\
& \quad \frac{\partial^{2} x(s, z)}{\partial s^{2}}-K(s)[1+\Delta k(z)] x= \\
& \frac{W_{T 0} C}{\gamma_{0}} \int_{0}^{z} \rho\left(z^{*}\right)\left(z-z^{*}\right) x\left(s, z^{*}\right) d z^{*}+ \\
& +K(s)[1+\Delta k(z)] x_{Q}(s)
\end{aligned}
$$

The initial conditions are:

$$
\begin{aligned}
& x(0, z)=0 \\
& \frac{\partial x}{\partial s}(s=0)=0
\end{aligned}
$$

The independent variables $s$ and $z$ represent the distance along the linac and the coordinate inside the bunch. $z$ is zero at the head and equal to the bunch length $l_{B}$ at the tail of the truncated bunch. The unknowns $\gamma(s, z)$ and $x$ are the energy Lorentz factor as well as the vertical transverse displacement along the bunch at a given linac position. A piecewise constant energy of the bunch along the different linac sectors is assumed so that no acceleration term proportional to $\frac{\partial x}{\partial s}$ appears in the equation of motion. In addition, a linear variation of the wakefield level along the bunch in both planes is assumed. While $W_{L 0}$ and $W_{L 1}$ represent the longitudinal wakefield $\left(W_{L}\right)$ at the head and tail of the bunch, $W_{T 0}$ stands for the transverse wakefield $\left(W_{T}\right)$ at the tail. We use a 4-th order Chebyshev expansion of a normalized Gaussian charge distribution in the range of $\pm 2 \sigma_{z}$ given by:

$$
\rho(z) \simeq \frac{75}{46 l_{B}}\left[\frac{1}{20}\left(\frac{4 z}{l_{B}}-2\right)^{4}-\frac{41}{100}\left(\frac{4 z}{l_{B}}-2\right)^{2}+1\right]
$$

The quantity $\Delta k(z)$ in Eq. (2) represents a variation of the focusing force inside the bunch which is caused by the energy dependent focusing (chromatic effect) as well as by the application of RF quadrupoles in order to reduce the emittance blowup caused by the presence of wakefields. The constant $C$ is defined as $C=4 \pi \epsilon_{0} r_{e} N$ where $\epsilon_{0}$ is the permittivity of free space, $N$ the number of particles in the bunch and $r_{e}$ the classical electron radius. The function $x_{Q}(s)$ represents the actual quadrupole misalignments as function of the position $s$. This function is either random or given by recurrence relations representing a trajectory correction [2].

\section{SOLUTION OF THE LONGITUDINAL EQUATION}

Eq. (2) can be solved in a straightforward way by simple integration w.r.t the independent variable $s$. This yields

$$
\begin{aligned}
& \gamma(s, z)=\gamma_{0}+\frac{e U s}{m_{0} c^{2}} \cos \left(k_{R F} z-\bar{\Phi}_{R F}\right)- \\
& -4 \pi \epsilon_{0} r_{e} N s R(z)
\end{aligned}
$$

with

$$
R(z)=\int_{0}^{z} W_{L}\left(z-z^{*}\right) \rho\left(z^{*}\right) d z^{*}
$$

In this notation, $\Phi_{R F}$ is the acceleration phase at the head of the bunch. The function $R(z)$ can easily be found by using the linear variation of $W_{L}$ inside a single bunch and the Chebyshev model (5) for the gaussian distribution. 
With the definition $\zeta=\frac{z}{l_{B}}, R(z)$ is given by

$$
\begin{aligned}
& R(z)= \\
& W_{L 0} \times \\
& \times\left(-\frac{16}{23} \zeta^{6}+\frac{144}{23} \zeta^{5}-\frac{559}{46} \zeta^{4}+\frac{157}{23} \zeta^{3}+\frac{6}{23} \zeta\right)+ \\
& +W_{L 1} \times \\
& \times\left(\frac{16}{23} \zeta^{6}-\frac{48}{23} \zeta^{5}+\frac{79}{46} \zeta^{4}+\frac{1}{23} \zeta^{3}+\frac{3}{23} \zeta^{2}\right)
\end{aligned}
$$

The energy spread $\delta(s, z)$ inside the bunch is defined in the usual way as $\delta(s, z)=(\gamma(s, z)-\gamma(s, 0)) / \gamma(s, z)$. However, as a simplification, only the asymptotic limit given as $\delta(z)=\lim _{s \rightarrow \infty} \delta(s, z)$ is used. In this way the $z$ - dependent detuning force $\Delta k(z)$ is given by

$$
\Delta k(z)=-\delta(z)+\alpha_{0} \sin \left(k_{R F} z-\bar{\Phi}_{R F Q}\right)
$$

where $\alpha_{0}$ is the maximum focusing strength of the RF quadrupole and $\bar{\Phi}_{R F Q}$ its phase at the head of the bunch.

\section{SOLUTION OF THE TRANSVERSE EQUATION}

The linear partial integro-differential equation for the transverse bunch displacement (2) can be solved in an easier way by separating two types of solution, one only depending on $s$ and one on both independent variables, $s$ and $z$. Hence, the solution is decomposed as

$$
x(s, z)=X(s)+y(s, z)
$$

and two new equations are obtained, one for the coherent part (independent of $z$ ) and one for the incoherent part (depending on $s$ and $z$ ) of the bunch oscillation:

$$
\begin{gathered}
\frac{d^{2} X(s)}{d s^{2}}-K(s) X(s)=K(s) x_{Q}(s) \\
\frac{\partial^{2} y(s, z)}{\partial s^{2}}-K(s)[1+\Delta k(z)] y(s, z)=X(s) \times \\
\times\left[K(s) \Delta k(z)+\frac{C W_{T 0}}{\gamma_{0} l_{B}} \int_{0}^{z} \rho\left(z^{*}\right)\left(z-z^{*}\right) d z^{*}\right]+ \\
+\frac{C W_{T 0}}{\gamma_{0} l_{B}} \int_{0}^{z} \rho\left(z^{*}\right)\left(z-z^{*}\right) y\left(s, z^{*}\right) d z^{*}+ \\
+K(s) \Delta k(z) x_{Q}(s)
\end{gathered}
$$

While Eq. (11) represents the betatron motion in the absence of wakefields and acceleration, Eq. (12) contains chromatic contributions as well as terms responsable for creating dispersion induced by the quadrupole misalignments. The weak focusing approximation is now introduced with the average tune defined by $\bar{q}=1 / \bar{\beta}$. However, this is used in the focusing forces but not in the terms containing the quadrupole misalignments, to keep their discrete nature. Computing the $\beta$ functions from the transfer matrices gives the dependence of $\bar{q}$

$$
\begin{aligned}
& \bar{q}(z)=\frac{3(1+\Delta k(z))}{L\left(3-(1+\Delta k(z))^{2} \sin ^{2} \frac{\mu}{2}\right)} \times \\
& \times \sqrt{(1-\cos \mu)\left[2-(1+\Delta k(z))^{2}(1-\cos \mu)\right]}
\end{aligned}
$$

In the case of the coherent equation (11) the tune follows by substituting $\Delta k(z)=0$ in the above equation [4],

$$
q=\left[L\left(\cot \frac{\mu_{\text {cell }}}{2}+\frac{2}{3} \tan \frac{\mu_{\text {cell }}}{2}\right)\right]^{-1} .
$$

In this way, the actual equations of motion become

$$
\begin{aligned}
& \frac{d^{2} X}{d s^{2}}+q^{2} X=K(s) x_{Q}(s) \\
& \frac{\partial^{2} y}{\partial s^{2}}+\bar{q}^{2}(z) y=X(s) \times \\
& \times\left[\frac{C W_{T 0}}{\gamma_{0} l_{B}} \int_{0}^{z} \rho\left(z^{*}\right)\left(z-z^{*}\right) d z^{*}-\left(q^{2}-\bar{q}^{2}(z)\right)\right]+ \\
& +\frac{C W_{T 0}}{\gamma_{0} l_{B}} \int_{0}^{z} \rho\left(z^{*}\right)\left(z-z^{*}\right) y\left(s, z^{*}\right) d z^{*}+ \\
& +K(s) \Delta k(z) x_{Q}(s) .
\end{aligned}
$$

\subsection{Solution for the coherent motion}

The coherent equation (15) describing the betatron motion in the absence of wakefields and under the influence of quadrupole misalignments can be solved by assuming localized kicks for the actual quadrupole displacements. In between these kicks the solution is sinusoidal and its amplitude is defined by the effects of the kicks upstream of the position considered. In order to describe this limitation to upstream quadrupoles, the Heaviside Function $H\left(s-s_{k}\right)$ is used which has the property to be strictly zero for negative arguments and equal to unity for positive ones. Here $s_{k}$ denotes the $k$-th quadrupole position given by $s_{k}=k L$ if $L$ is half a FODO period. In addition to this causality principle, the method of superposition of all the solutions due to single kicks is used. This is justified by the linearity of the equation and the use of thin lenses. The solution is then:

$$
X=\frac{2}{q L} \sin \frac{\mu}{2} \sum_{k}(-1)^{k} x_{k} \sin \left[q\left(s-s_{k}\right)\right] H\left(s-s_{k}\right)
$$

The $x_{k}$ are the actual quadrupole misalignements at position $s_{k}$. In Fig. 1, a typical solution is shown for $X$ in a FODO lattice with misaligned quadrupoles with an RMS displacement of $10 \mu \mathrm{m}$, when $\mu=105^{\circ}$. The resonant behaviour of the solution arises from the continuity of the frequency spectrum of a random function which contains the unperturbed betatron frequency on the left side of Eq. (15). 


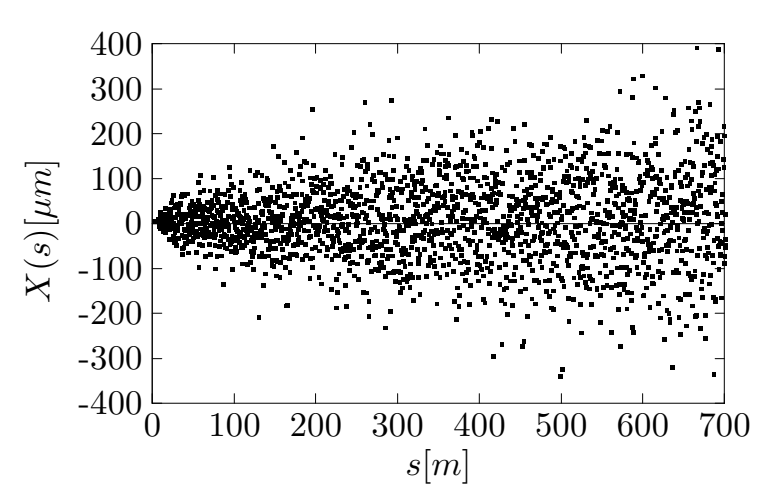

Fig. 1 Solution $X(s)$ in the presence of quadrupole misalignments

\subsection{Solution for the incoherent motion}

Equation (16) can be rewritten as

$$
\begin{aligned}
& \frac{\partial^{2} y}{\partial s^{2}}+\bar{q}^{2}(z ; \mathbf{E} \Delta k(z)) y=A(z) X(s)+ \\
& +K(s) \Delta k(z) x_{Q}(s)+ \\
& +\epsilon \frac{C W_{T 0}}{\gamma_{0} l_{B}} \int_{0}^{z} \rho\left(z^{*}\right)\left(z-z^{*}\right) y\left(s, z^{*}\right) d z^{*}
\end{aligned}
$$

with

$$
A(z)=\left[\frac{C W_{T 0}}{\gamma_{0} l_{B}} \int_{0}^{z} \rho\left(z^{*}\right)\left(z-z^{*}\right) d z^{*}-\left(q^{2}-\bar{q}^{2}(z)\right)\right]
$$

Using the approximation (5) for $\rho(z)$ the coefficient function $A(z)$ becomes a sixth order polynomial. The formal perturbation parameters $E$ and $\epsilon$ multiply small contributions such as the wakefields. However, the expansion is only made with respect to one of them, namely $\epsilon$ in order to keep the $z$ dependent tune $\bar{q}$ to any order and in this way avoid the occurence of secular terms. Hence $y=y^{(0)}(s, z ; E)+\epsilon y^{(1)}(s, z ; E)+O\left(\epsilon^{2}\right)$. Already the lowest order contribution $y^{(0)}(s, z)$ has been proven to describe the solution sufficiently well for the case of the CLIC main linac. The equation for $y^{(0)}$ is

$$
\frac{\partial^{2} y^{(0)}}{\partial s^{2}}+\bar{q}^{2}(z) y^{(0)}=X(s) A(z)+K(s) x_{Q}(s) \Delta k(z)
$$

and this linear inhomogeneous second order equation is solved as usual by adding the homogeneous solution $y_{h}$ to the particular one found applying Green's formula.

$$
\begin{aligned}
& y^{(0)}(s, z)=y_{h}+\frac{\sin \bar{q} s}{\bar{q}} \int_{0}^{s} \cos \bar{q} s^{*} g\left(s^{*}\right) d s^{*}- \\
& -\frac{\cos \bar{q} s}{\bar{q}} \int_{0}^{s} \sin \bar{q} s^{*} g\left(s^{*}\right) d s^{*}
\end{aligned}
$$

where $g(s)$ represents the right hand side of Eq. (20). After integration the final result becomes

$$
\begin{aligned}
& y^{(0)}(s, z)=y_{0} \cos \bar{q}(z) s+\frac{y_{0}^{\prime}}{\bar{q}(z)} \sin \bar{q}(z) s+ \\
& +A(z) \sum_{k=1}^{N_{Q}} \frac{2}{L q \bar{q}(z)} \sin (\mu / 2)(-1)^{k} x_{k} H\left(s-s_{k}\right) \times
\end{aligned}
$$

$$
\begin{aligned}
& \times\left[\sin \bar{q}(z) s I_{1}\left(s, s_{k}\right)-\cos \bar{q}(z) s I_{2}\left(s, s_{k}\right)\right]+ \\
& +\Delta k(z) \frac{2}{L \bar{q}(z)} \sin (\mu / 2) \times \\
& \times \sum_{k=1}^{N_{Q}}(-1)^{k} x_{k} \sin \bar{q}(z)\left(s-s_{k}\right) H\left(s-s_{k}\right)
\end{aligned}
$$

where

$$
\begin{aligned}
& I_{1}\left(s, s_{k}\right)=\int_{s_{k}}^{s} \cos \bar{q} s^{*} \sin q\left(s-s_{k}\right) d s^{*} \\
& I_{2}\left(s, s_{k}\right)=\int_{s_{k}}^{s} \sin \bar{q} s^{*} \sin q\left(s-s_{k}\right) d s^{*} .
\end{aligned}
$$

Evidently $I_{1,2}$ permit a closed form solution and these expressions contain denominators of the form $q^{2}-\bar{q}^{2}(z)$ which clearly exhibit the near resonance dynamics of the single bunch problem with wakefields.

Fig. 2 shows a comparison of the perturbative analytical solution $y^{(0)}$ to a numerical one obtained by the code MUSTAFA [5] for CLIC main linac parameters of $N=6 \cdot 10^{9}$ and an RMS bunch length of $\sigma_{z}=200 \mu \mathrm{m}$ at the linac position $s=1000 \mathrm{~m}$.

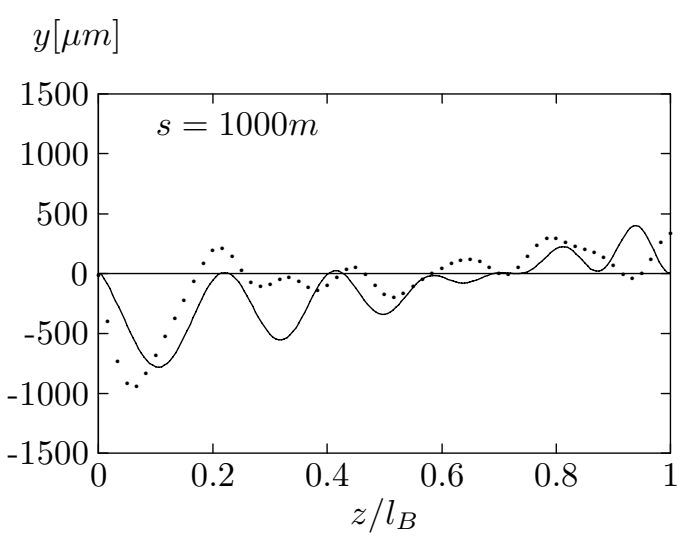

Fig.2 Comparison of the analytical and the numerical result for $y(z)$ at $s=1000 \mathrm{~m}$.

To conclude, it can be pointed out that the agreement in amplitude and frequency of the analytical (full line) and numerical result (points) is very good. The local differences in phase mainly originate from the fact that MUSTAFA uses a strong focusing model while the theory is based on the weak focusing approximation. In addition the sequence of random numbers chosen in both examples is different although both sequences have the same RMS value.

\section{REFERENCES}

[1] G. Guignard, J. Hagel, CERN-SL-98-015 AP (1998)

[2] G. Guignard, J. Hagel, CERN-SL-99-006 AP (1999), 'Theory of Single Bunch Stability and Dynamics in Linacs with Strong Wakefields and Misalignments, submitted to Nuclear Instruments and Methods in Physics Research A.

[3] G.V. Stupakov, SLAC-AP-108 (1997)

[4] T. Raubenheimer, CLIC Note 347 (1997)

[5] G. Guignard, J. Hagel, CERN-SL-98-002 AP (1998) 